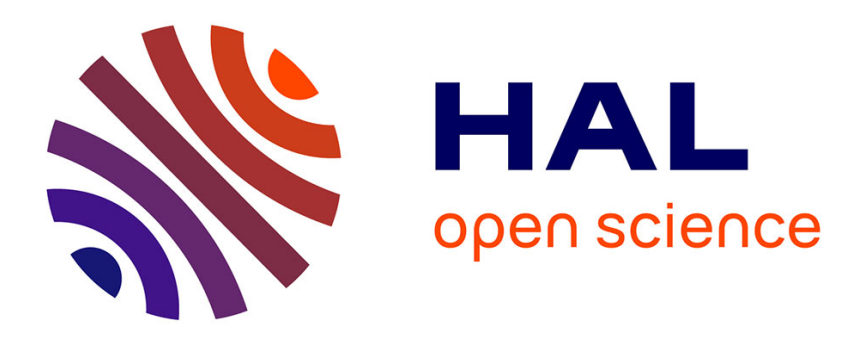

\title{
Pour une politique agricole commune ambitieuse, mais renouvelée
}

\author{
Marion Guillou
}

\section{To cite this version:}

Marion Guillou. Pour une politique agricole commune ambitieuse, mais renouvelée. Politique étrangère, 2011, 2, pp.321-334. 10.3917/pe.112.0321 . hal-02643308

\section{HAL Id: hal-02643308 \\ https://hal.inrae.fr/hal-02643308}

Submitted on 28 May 2020

HAL is a multi-disciplinary open access archive for the deposit and dissemination of scientific research documents, whether they are published or not. The documents may come from teaching and research institutions in France or abroad, or from public or private research centers.
L'archive ouverte pluridisciplinaire HAL, est destinée au dépôt et à la diffusion de documents scientifiques de niveau recherche, publiés ou non, émanant des établissements d'enseignement et de recherche français ou étrangers, des laboratoires publics ou privés. 


\title{
POUR UNE POLITIQUE AGRICOLE COMMUNE AMBITIEUSE, MAIS RENOUVELÉE
}

\author{
Marion Guillou
}

I.F.R.I. | Politique étrangère

2011/2 - Eté

pages 321 à 334

ISSN 0032-342X

Article disponible en ligne à l'adresse:

http://www.cairn.info/revue-politique-etrangere-2011-2-page-321.htm

Pour citer cet article :

Guillou Marion, «Pour une Politique agricole commune ambitieuse, mais renouvelée », Politique étrangère, 2011/2 Eté, p. 321-334. DOI : 10.3917/pe.112.0321

Distribution électronique Cairn.info pour I.F.R.I..

(C) I.F.R.I.. Tous droits réservés pour tous pays.

La reproduction ou représentation de cet article, notamment par photocopie, n'est autorisée que dans les limites des conditions générales d'utilisation du site ou, le cas échéant, des conditions générales de la licence souscrite par votre établissement. Toute autre reproduction ou représentation, en tout ou partie, sous quelque forme et de quelque manière que ce soit, est interdite sauf accord préalable et écrit de l'éditeur, en dehors des cas prévus par la législation en vigueur en France. II est précisé que son stockage dans une base de données est également interdit. 


\section{page 319 dossier}

\section{L'AVENIR DE LA PAC}




\title{
Pour une Politique agricole commune ambitieuse, mais renouvelée
}

\author{
Par Marion Guillou
}

Marion Guillou est présidente de l'Institut national de la recherche agronomique (INRA) et présidente d'Agreenium. Elle est également membre de plusieurs commissions d'experts et académies en France et à l'étranger.

Plusieurs facteurs, dont les évolutions démographiques, climatiques et environnementales, obligent à repenser la Politique agricole commune (PAC). Son renouvellement doit conduire à une stabilisation des revenus des agriculteurs et des prix des produits agricoles, à une meilleure prise en compte des biens publics que sont notamment le climat et la biodiversité et à un développement de l'innovation « verte ».

politique étrangère

Les discussions sur la Politique agricole commune (PAC) de l'après-2013 vont bientôt sortir de l'étape préliminaire pour entrer dans une phase plus difficile, où chaque détail aura son importance. Il ne faudra pas perdre de vue l'essentiel : élaborer la PAC de demain, c'est construire l'agriculture européenne d'après-demain. Ceci alors que l'agriculture mondiale est confrontée à un contexte historique inédit. Elle paraît en effet à nouveau essentielle pour l'avenir de nos sociétés, après avoir été considérée depuis 30 ans comme une activité de second rang. Le rapport de la Banque mondiale de 2008 reconnaît ainsi l'agriculture comme un facteur essentiel du développement. Les émeutes dites de la faim de 2008 furent certes des émeutes urbaines, liées pour l'essentiel aux difficultés d'accès à la nourriture. Mais ce serait une grave erreur d'oublier que les personnes souffrant de la faim sont majoritairement des agriculteurs : le développement agricole et rural est un facteur clé de sortie de la pauvreté et de la sous-alimentation dans le monde.

L'agriculture n'est pas accessoire. Les évolutions démographiques, conjuguées aux changements globaux, à la raréfaction des énergies fossiles et à la dégradation des ressources naturelles, appellent l'agriculture à produire plus, à produire d'autres choses et à produire autrement. 
Au cours des dernières années, de multiples travaux ont été conduits pour dessiner des trajectoires possibles et en inférer les voies à préparer dès aujourd'hui. Le Millenium Ecosystem Assessment (MEA) ${ }^{1}$ a ouvert le ban au début des années 2000. En France, l'Institut national de la recherche agronomique (INRA) et le Centre de coopération internationale en recherche agronomique pour le développement (CIRAD) ont engagé en 2006 une prospective sur les systèmes alimentaires et agricoles mondiaux à l'horizon 2050, intitulée Agrimonde ${ }^{2}$. En 2008, le RoyaumeUni, traditionnellement confiant dans l'idée que les échanges internationaux pouvaient assurer leur sécurité alimentaire et celle de la planète, ont également lancé une prospective, Global Food and Farming Futures ${ }^{3}$. Ils concluent tous au nécessaire réinvestissement dans l'agriculture et la recherche agronomique.

\section{Les défis auxquels fait face l'agriculture}

$\mathrm{Au}$ risque de rappeler quelques constats aujourd'hui bien connus, résumons les principales évolutions qui vont marquer le paysage mondial sur les prochaines décennies.

1 - Tout d'abord l'évolution démographique. L'estimation médiane pour 2050 est d'environ 9 milliards d'habitants sur Terre. Cette évolution se double d'une transition alimentaire. En particulier, la consommation de produits animaux augmente fortement quand le produit intérieur brut (PIB) croît, puis se ralentit et enfin se tasse dans les sociétés les plus riches.

2 - Une autre évolution majeure est l'augmentation d'une demande de produits agricoles pour des débouchés non alimentaires. Différents facteurs y contribuent: une augmentation de la demande en mobilité dans les sociétés qui deviennent plus riches, la raréfaction et le renchérissement des ressources fossiles, la demande de substitution de produits issus des ressources fossiles par des produits renouvelables et moins polluants, etc. L'agriculture est donc appelée à répondre à une demande croissante de carbone végétal destiné à la production de bioénergies et de bioproduits.

1. MEA, Global Assessment Reports, vol. 1 - Current State \& Trends ; vol. 2 - Scenarios ; vol. 3 - Policy Responses, Washington, DC, Island Press, 2005.

2. S. Paillard, S. Treyer et B. Dorin (dir.), Agrimonde. Scénarios et défis pour nourrir le monde en 2050, Paris, Éditions Quæ, 2010.

3. UK Government, Department for Business, Innovation and Skills, Global Food and Farming Futures, Londres, 2001, disponible à l'adresse suivante : <http://www.bis.gov.uk/foresight/our-work/projects/ current-projects/global-food-and-farming-futures 2011>. 
3 - Le changement climatique : les nombreux travaux scientifiques dont le Groupe d'experts intergouvernemental sur l'évolution du climat (GIEC) fait régulièrement la synthèse mettent en évidence un processus de réchauffement climatique lié à l'augmentation des émissions de gaz à effet de serre (GES). L'enjeu est de contenir ce réchauffement et de s'y adapter. L'agriculture est une des activités les plus concernées, comme émetteur important de GES mais aussi comme puits de carbone dans les sols et les prairies. Elle est aussi une activité particulièrement vulnérable au climat. En France, des scientifiques de l'INRA et d'Arvalis ont analysé l'évolution des rendements du blét $;$ ils ont mis en évidence un effet négatif du climat depuis 1990 environ, aggravé à partir de 1999 par des facteurs agronomiques; le progrès génétique a cependant permis de maintenir les rendements à un niveau stable. Un article récemment paru dans Science ${ }^{5}$ confirme l'effet négatif déjà perceptible du changement climatique sur les rendements mondiaux du maïs et du blé.

4 - Une dégradation des ressources naturelles qui se traduit par une perte de biodiversité, une baisse de la fertilité des sols et une dégradation quantitative ou qualitative des ressources en eau. Au plan national, les connaissances scientifiques disponibles montrent qu'il y a une diminution de la biodiversité dans les espaces agricoles sur les 50 dernières années $^{6}$. À l'échelle de la planète, les estimations du rythme actuel d'extinction des espèces le situent à un niveau 100 à 1000 fois supérieur à son niveau «normal».

5 - Une mondialisation des échanges et des risques : la circulation de personnes et de biens a été largement multipliée depuis un siècle. Elle conduit à une circulation plus rapide des agents pathogènes et à une augmentation exponentielle des invasions biologiques : l'analyse de 1300 publications pilotée par des scientifiques de l'INRA a ainsi montré que les invasions biologiques de vertébrés ont augmenté de moins d'une invasion par siècle entre - 9200 et 1600, à 136 au cours des 50 dernières années ${ }^{7}$.

Ces tendances lourdes se conjuguent pour dessiner un triple défi pour l'agriculture du $\mathrm{XXI}^{\mathrm{e}}$ siècle : démographique, énergétique et environnemental. Pour y faire face, il faudra augmenter la production agricole, tout

4. N. Brisson, P. Gate, D. Gouache, G. Charmet, F.-X. Oury et F. Huard, "Why Are Wheat Yields Stagnating in Europe? A Comprehensive Data Analysis for France ", Field Crops Research, vol. 119, $\mathrm{n}^{\circ} 1,2010$, p. 201-212.

5. D. B. Lobell et alii, « Climate Trends and Global Crop Production Since 1980 », Science, 5 mai 2011. 6. X. Le Roux, R. Barbault, J. Baudry, F. Burel, I. Doussan, E. Garnier, F. Herzog, S. Lavorel, R. Lifran, J. Roger-Estrade, J.-P. Sarthou et M. Trommetter (dir.), Agriculture et biodiversité. Valoriser les synergies, Expertise scientifique collective, synthèse du rapport, Paris, INRA, 2008.

7. M. Pascal, O. Lorvelec et J.-D. Vigne, Invasions biologiques et extinctions. 11000 ans d'histoire des vertébrés en France, Paris, Belin/Éditions Quæ, 2006. 
en consommant moins d'énergie et en respectant mieux l'environnement. En d'autres termes, il faudra développer une agriculture productive, économe et écologique via l'innovation et l'investissement. Il faudra simultanément agir sur les régimes alimentaires, car ceux des pays occidentalisés ne sont pas généralisables à toute la planète, et réduire les pertes et gaspillages, du champ de l'agriculteur à l'assiette du consommateur.

\section{Le renouvellement de la PAC}

Dans un contexte porteur pour l'agriculture, la tentation pourrait être de réduire les ambitions et les moyens de la PAC. La prospective Agriculture $2013^{8}$ avait mis en évidence qu'une croissance mondiale soutenue, ou une politique ambitieuse de promotion des biocarburants en Europe et aux États-Unis, ou encore l'aboutissement des négociations agricoles du cycle de Doha à l'Organisation mondiale du commerce (OMC) pourraient avoir sur les prix et les revenus agricoles des effets d'une ampleur équivalente à ceux des soutiens octroyés via la PAC : effets à la hausse pour la croissance et la demande en biocarburants, effets à la baisse pour les accords à l'OMC.

En l'absence de subventions, les exploitations agricoles françaises auraient un revenu proche de zéro

En conclure que nous pouvons nous passer d'une PAC forte et ambitieuse serait néanmoins une erreur. Le contexte à moyen et long terme est certes porteur. Il est contraignant sur le double plan énergétique et environnemental. Il est aussi instable, comme les épisodes récents de hausse brutale des cours agricoles et de baisse aussi rapide l'illustrent. Et cette instabilité ira en augmentant compte tenu du changement climatique et de la mondialisation des économies. En outre, renoncer à une PAC forte mettrait à mal une grande partie des exploitations agricoles communautaires qui, confrontées à la concurrence d'agricultures étrangères plus compétitives en coûts et très souvent moins contraintes sur le plan environnemental, sanitaire et/ou social, ne sont viables que grâce aux aides de la PAC. Enfin, cette politique a un rôle majeur à jouer en orientant le progrès technique et l'innovation en faveur de systèmes agricoles productifs, économes et écologiques.

\section{Préserver la viabilité des exploitations}

On ne le sait sans doute pas assez: en l'absence de subventions, dans le contexte de prix des produits agricoles et des intrants des dernières années, les exploitations agricoles françaises auraient, en moyenne, un revenu

8. H. Guyomard, C. Le Mouël (dir.), C. Jez, A. Forslund et E. Fournel, Prospective “ Agriculture 2013 ». Résultats et enseignements principaux par scénario, Paris, INRA, 2007. 
proche de zéro. Comme le soulignent Hervé Guyomard et Vincent Chatellier ${ }^{9}$, sur la période 2007-2009, les aides directes reçues au titre des deux piliers de la PAC ont représenté $20 \%$ du chiffre d'affaires de la ferme France (champ des exploitations dites professionnelles) et $90 \%$ de son résultat courant avant impôt (30 200 euros par exploitation et 20900 euros par emploi agricole familial). Mais même avec ces aides directes, le niveau de vie des ménages agricoles français reste inférieur, en moyenne - avec néanmoins de fortes disparités entre exploitations -, à celui de l'ensemble des ménages ${ }^{10}$. Rappelons aussi que le nombre d'exploitations agricoles diminue régulièrement en France (d'environ $3 \%$ par an).

La contribution à l'agriculture des consommateurs et des contribuables diminue elle aussi.

- La part du budget des ménages consacrée à l'alimentation est passée de $25 \%$ dans les années 1960 à $15 \%$ aujourd'hui, le coût des matières premières agricoles représentant en outre une part de plus en plus réduite de ce budget alimentaire.

- Depuis les années 1980, l'apport du contribuable à la PAC a baissé relativement à sa richesse, passant de $0,65 \%$ à $0,40 \%$ du PIB, cette réalité étant souvent occultée par le poids de la PAC dans le budget communautaire (un peu plus de $40 \%$ ), poids qui s'explique par le fait qu'il s'agit de la seule politique communautaire intégrée.

Ces chiffres plaident en faveur d'une PAC dotée à l'avenir de moyens financiers au moins équivalents à ceux de la période passée afin d'assurer dans la durée la viabilité des exploitations et de permettre aux agriculteurs de relever les défis rappelés plus haut. De tels flux financiers pourront choquer certains à l'heure où l'Europe et la France sont en crise économique et sociale, peinent face à la concurrence des pays émergents et que d'autres priorités légitimes exigent des ressources (éducation, recherche, emploi, etc.). Ces chiffres montrent néanmoins que les agriculteurs ne sont pas des privilégiés et qu'un transfert de richesse en faveur de l'agriculture - au demeurant très limité au regard de la richesse de l'Union européenne (UE) - est une démarche raisonnable pour sécuriser le présent et préparer l'avenir. Certes, l'absence de PAC n'aurait pas pour effet la disparition complète de l'agriculture. Elle entraînerait néanmoins un mouvement majeur de concentration de l'agriculture européenne dans les exploitations et les régions où elle est la plus rentable, avec une cohorte d'impacts

9. V. Chatellier et H. Guyomard, Le Budget de la PAC et le ciblage des aides, Ceras, revue Projet, $\mathrm{n}^{\circ} 321$, avril 2011.

10. D'après Les Revenus et le patrimoine des ménages, Paris, INSEE, 2010. Sources : INSEE, DGFIP, CNAF, CNAV, CCMSA, enquête Revenus fiscaux et sociaux 2007. 
négatifs sur l'emploi, la vie des territoires ruraux, l'entretien des espaces et les services environnementaux associés ${ }^{11}$. D'ailleurs, les pays développés, à l'exception de ceux de l'Océanie et dans une moindre mesure du Canada, soutiennent également leur agriculture : Corée du Sud, Japon, Norvège, Suisse, États-Unis, etc. La légitimité d'un budget agricole européen conséquent sera d'autant plus facilement reconnue et acceptée que les inégalités actuelles de sa distribution seront réduites et que l'argent ciblera la sécurité alimentaire, la protection de l'environnement et le développement des territoires ruraux.

Pour illustrer l'inégale répartition des aides agricoles, rappelons qu'à l'échelle de l'UE, 7,8 millions d'exploitations agricoles reçoivent des aides directes au titre du premier pilier de la PAC pour un montant annuel moyen d'environ 5000 euros, mais 30800 d'entre elles reçoivent plus de 100000 euros d'aides directes par an ${ }^{12}$.

En France, la mise en œuvre de la réforme de la PAC de 2008 dite du «bilan de santé » a permis d'atténuer les écarts entre exploitations par rééquilibrage en faveur des productions animales basées sur l'herbe au détriment essentiellement des productions céréalières et oléagineuses ${ }^{13}$. Toutefois, le niveau des aides inclut encore une forte composante historique: le montant des aides directes répond toujours à une logique ancienne de compensation de la baisse des prix garantis et continue à avantager les zones et les exploitations historiquement les plus productives. La question de la redistribution des aides au plan national est encore largement devant nous. À l'échelle européenne, la variabilité des aides en fonction de la taille de l'exploitation et de l'emploi occupé et l'égalité de traitement entre les États membres les plus anciens et les plus récents sont au cœur des débats. Cette redistribution entre pays et, à l'intérieur de chaque État membre, entre exploitations et entre productions, doit être réfléchie au regard des objectifs visés.

\section{PAC et stabilisation}

Tout autant que le soutien des revenus, la future PAC devra viser une relative stabilisation des prix et des marchés. Les fluctuations erratiques des prix mondiaux et européens observés depuis 2006 appellent des

\footnotetext{
11. A. Gohin, «Quelles conséquences d'une suppression de la PAC après 2013 ? ", Revue d'économie politique, vol. $119, \mathrm{n}^{\circ} 4,2009$.

12. D'après les statistiques de 2009 publiées par la direction générale de l'Agriculture et du Développement rural de la Commission européenne.

13. V. Chatellier et $\mathrm{H}$. Guyomard, «Le bilan de santé de la PAC et le rééquilibrage des soutiens à l'agriculture française ", Économie rurale, n 323, mai-juin 2010.
} 
mesures correctrices. Ceci dans un contexte où la volatilité devrait très vraisemblablement être la règle à l'avenir : le changement climatique, la financiarisation des marchés agricoles et les réactions désordonnées des États sur les marchés via des politiques d'interdiction des exportations ou de subventionnement des importations auront tendance à amplifier les fluctuations des cours, à la hausse comme à la baisse, et leurs effets contraires $^{14}$. À problème mondial, réponse mondiale. Mais tout en œuvrant sur la scène internationale pour mettre fin à la variabilité excessive des prix agricoles dont les premières victimes sont les agriculteurs et les consommateurs pauvres, l'Europe doit mettre en place des mécanismes internes dans le même objectif : limiter les fluctuations des cours et leurs conséquences négatives.

Les effets de cours élevés de céréales et d'oléagineux sont ambivalents : favorables en termes de revenus pour les producteurs de ces produits, ils ont clairement un impact négatif pour les éleveurs qui dépendent d'achats externes de céréales et de tourteaux d'oléagineux pour l'alimentation de leurs animaux et ne parviennent pas à répercuter, du moins pas en totalité, les surcoûts de production vers leur aval. Dans un régime opposé, les prix bas font souffrir les agriculteurs et menacent la viabilité des exploitations. Enfin, la volatilité des prix n'offre pas une visibilité de moyen terme suffisante pour encourager les investissements nécessaires à la transformation de l'agriculture.

Du côté des consommateurs, des prix très élevés empêchent l'accès à la nourriture et constituent un facteur premier d'insécurité, comme en témoignent les émeutes de la faim de 2008 ou les événements plus récents en Afrique du Nord et au Proche-Orient qui trouvent une part d'explication dans les cours élevés des biens agricoles et alimentaires.

Même si le problème est mondial, la solution ne saurait être le repli sur soi. Comme l'a montré la prospective Agrimonde, plusieurs grandes régions du monde resteront déficitaires en produits agricoles et alimentaires et par conséquent devront pouvoir recourir aux importations pour assurer la sécurité alimentaire de leurs populations : 1'Afrique, le Proche et Moyen-Orient et l'Asie auront demain un besoin accru d'importations agricoles en provenance des zones excédentaires, l'Amérique latine (plus particulièrement, l'Argentine et le Brésil), l'Amérique du Nord, l'Europe de l'Ouest et les pays de l'ex-Union soviétique.

14. H. Guyomard, «Expliquer les évolutions des cours des matières premières agricoles : à l'impossible nul n'est tenu! ", OCL, vol. 15, n 6, 2009. 
Les premières initiatives adoptées aux États-Unis et en Europe pour réguler les marchés financiers vont dans le bon sens. Elles visent à améliorer la transparence sur les transactions et à limiter les risques d'abus de

\section{Limiter les excès position sur les marchés. Cependant, même complé-} de volatilité des cours merciaux des «purs"spéculateurs et en limitant les agricoles capacités d'action de ces derniers, elles ne suffiront pas à limiter les excès de volatilité des cours agricoles et leurs conséquences délétères. Trois types d'action doivent simultanément être mobilisés au niveau mondial.

- La transparence sur l'état des stocks mondiaux et la constitution de stocks publics minimums avec un triple objectif: premièrement, une meilleure information sur les données de l'économie réelle qui limitera les risques d'emballement purement spéculatifs sur les marchés financiers; deuxièmement, une capacité d'anticipation et d'alerte pour coordonner des actions entre États; enfin, l'action en situation de crise par mise à disposition des quantités stockées pour ceux qui en ont le plus besoin. En outre, une telle coordination internationale du stockage agricole devrait permettre de briser un facteur de hausse brutale des cours agricoles qui surréagissent dès lors que les stocks sont inférieurs à des niveaux «planchers ».

- Des outils économiques «internes » pour les pays et les zones qui le souhaitent, permettant de lisser les effets de la volatilité des cours sur les revenus des agriculteurs (dispositifs contracycliques, assurances, etc.) ou sur l'accès à l'alimentation pour les consommateurs (bons d'achat, etc.).

- Un engagement multilatéral à ne pas interdire les exportations de façon unilatérale.

Ces actions supposent une gouvernance internationale résolue autour des enjeux de la sécurité alimentaire mondiale. L'Europe doit peser de tout son poids pour qu'une telle gouvernance se mette en place, au risque de voir se multiplier les épisodes d'émeutes de la faim et de voir grandir le nombre de personnes en situation de sous-alimentation et de pauvreté. L'Europe y gagnerait également en termes d'efficacité des instruments de stabilisation de la PAC, qu'il s'agisse des filets de sécurité à activer en situation de crise ou des mécanismes de protection contre les effets négatifs de l'instabilité. Ces mécanismes nécessitent un soutien public de façon à ce que tous les agriculteurs puissent en bénéficier.

Plus généralement, l'instabilité appelle un renouvellement des approches en matière de commerce international agricole et alimentaire et des règles 
qui le régissent. Il faut remettre le droit à la sécurité alimentaire au centre des négociations et reconnaître que bon nombre de pays pauvres qui ne disposent d'autres ressources que celles de l'agriculture ont un droit légitime à soutenir cette dernière pour assurer les bases de leur développement; leur accorder un traitement spécial et différencié à l'OMC ne suffit pas. Cela ne signifie pas qu'il faille renoncer à un commerce agricole mondial plus transparent et ouvert, mais les accords internationaux en ce domaine, qu'ils soient multilatéraux ou bilatéraux, doivent mieux intégrer les dimensions sociales et environnementales, celles-ci ne pouvant être laissées aux lois du seul marché.

\section{Légitimité de la PAC et biens publics}

L'agriculture est à la fois productrice et utilisatrice de nombreux biens publics, biens que la théorie économique définit à partir de deux critères : la non-rivalité (la consommation du bien par un agent économique ne diminue pas la quantité que les autres peuvent consommer) et la nonexclusion (impossibilité d'exclure un agent de l'accès au bien), ces deux critères pouvant être partiellement remplis. Citons, parmi les biens publics utilisés et produits par l'agriculture, la stabilité du climat, la biodiversité, la qualité de l'eau ou encore la cohésion sociale. La recherche en économie publique montre clairement que le seul marché, via les ajustements entre l'offre et la demande des différentes marchandises, ne permet pas d'assurer une fourniture de ces biens publics aux niveaux désirés par la société. À l'extrême, le libre jeu des acteurs économiques peut entraîner ce qu'on appelle la «tragédie des communs »: la dégradation irréversible de ressources naturelles dans le cadre d'usages individuels non coordonnés. L'intervention des pouvoirs publics est donc requise pour agréger les demandes des différents biens publics, identifier les niveaux optimums de fourniture - étape difficile qui est souvent remplacée par des concertations et des compromis, reflets des diverses demandes sociales - et compléter le marché par des signaux, contraignants et/ou incitatifs (réglementations, normes, quotas, jeu de taxes et subventions, etc.), qui orienteront les choix des acteurs dans le sens désiré.

Cette ambition en matière de biens publics doit être au cœur de la PAC de l'après-2013. L'attention doit tout particulièrement se porter sur les biens environnementaux : stabilité du climat, biodiversité, eau et sol. Non seulement parce que ces biens publics environnementaux correspondent à une nécessité pour la société de demain et sont un facteur clé d'attractivité des territoires ruraux, mais aussi parce qu'ils sont essentiels pour l'agriculture qui occupe et valorise près de $60 \%$ du territoire français métropolitain, et un peu moins du territoire européen. 
Jusqu'à ce jour, les mesures de la PAC à visée environnementale sont restées trop modestes au regard des enjeux. Les mesures agri-environnementales (MAE) ne représentent ainsi qu'environ $10 \%$ du budget de la PAC et la conditionnalité des aides du premier pilier, c'est-à-dire la subordination de leur octroi au respect de règlements et de bonnes pratiques environnementales, est encore trop limitée. Les MAE font l'objet d'évaluations assez critiques sur plusieurs plans: un surcroît disproportionné de charges administratives, un impact limité sur l'environnement $\mathrm{du}$ fait de la dispersion spatiale des mesures, des contrats de trop courte durée, des incitations économiques trop faibles, etc. Ces défauts sont facilement corrigeables pour peu que la volonté politique soit au rendez-vous. Plus importants sont

Les mesures de la PAC les deux points suivants. En premier lieu, à visée environnementale la nécessité d'accorder davantage d'attention à la biodiversité et au changement climatique (dans les deux dimensions de contribution de l'agriculture à la réduction des émissions de GES et d'adaptation du secteur agricole aux effets du changement climatique). Il s'agit là de deux biens publics globaux trop négligés jusqu'à présent, chaque État membre ayant un intérêt égoïste à adopter un comportement de "passager clandestin» en privilégiant des biens publics plus locaux. Dans cette perspective, n'ayons pas peur d'inclure l'agriculture dans les marchés de $\mathrm{CO}_{2}$ et de biodiversité de façon à pallier l'insuffisance des ressources budgétaires agricoles. En deuxième lieu, la nécessité de rémunérer les contributions environnementales au-delà de la logique actuelle des MAE, qui compense les surcoûts pour l'agriculture.

La conditionnalité des aides directes du premier pilier peut constituer une politique environnementale de plus grande ampleur par l'importance du public touché. Déconnectée d'une estimation de la valeur sociale des externalités visées et associée à des modalités d'application faiblement incitatives, elle n'a pas encore convaincu de son efficacité la Cour des comptes européenne et de nombreux économistes. Cela tient pour partie au fait que les dispositions sont encore trop modestes pour être efficaces. La critique ne doit pas être prétexte à l'abandonner. Le verdissement du premier pilier doit être mis à profit pour conditionner le soutien budgétaire au respect d'exigences minimales en matière de pratiques et de systèmes de culture et d'élevage économes et écologiques. Au-delà de ces minima, place aux MAE et aux marchés environnementaux pour rémunérer les efforts additionnels. 


\section{La future PAC et l'innovation « verte »}

Nourrir la planète à l'horizon 2050 de façon durable suppose des évolutions profondes des pratiques et des systèmes agricoles et alimentaires, en Europe comme ailleurs dans le monde. Il faudra, compte tenu des disponibilités limitées en terres et des autres usages concurrents, augmenter les rendements, même si l'Organisation de coopération et de développement économiques (OCDE) n'est pas la zone où la croissance la plus forte est attendue dans la mesure où les rendements y sont déjà élevés et montrent un certain essoufflement depuis les années 1980. Il faudra s'adapter à de nouvelles conditions climatiques, concevoir des systèmes agricoles résilients et trouver de nouvelles voies à la fois productives, économes et écologiques. Dans cette perspective, les systèmes agricoles européens sont trop dépendants d'intrants énergétiques et chimiques (engrais et pesticides), certes facteurs de compétitivité et de sécurité pour les agriculteurs, mais également sources de coûts et de pollutions. Les attentes de la société pour une agriculture sobre en intrants et respectueuse de l'environnement sont claires. Au niveau européen, à travers différentes réglementations (" paquet pesticides », directive-cadre sur l'eau, directive Habitats, etc.), ou à l'échelle nationale dans le cadre notamment du Grenelle de l'environnement, des mesures sont promues pour faire évoluer les pratiques agricoles. Le plan Ecophyto 2018 qui vise ainsi une réduction de l'emploi des pesticides de $50 \%$ «si possible » d'ici 2018 nécessite des évolutions. L'innovation doit être le troisième objectif de la PAC de demain.

L'innovation «verte » ne saurait découler des seuls signaux adressés à l'agriculture par les politiques environnementales et la composante environnementale de la PAC, même révisée selon la logique développée ci-dessus. Dans le récent rapport du Conseil économique pour le développement durable relatif au financement de la croissance verte ${ }^{15}$, Patricia Crifo souligne la nécessité de compléter les politiques à visée environnementale par des politiques centrées sur l'innovation. Plusieurs caractéristiques de l'innovation verte génèrent un besoin spécifique d'intervention publique: des effets d'apprentissage importants avant diffusion et adoption à large échelle, des innovations complexes et cumulatives qui rendent plus difficile l'appropriation des bénéfices, des incertitudes sur les perspectives futures par comparaison aux technologies déjà en place. Politiques de l'environnement et politiques de l'innovation doivent être combinées pour renforcer leurs effets propres et générer les conditions

15. Conseil économique pour le développement durable, Le Financement de la croissance verte, Paris, La Documentation française, 2011. 
favorables à une croissance verte. Elles seront utilement complétées par des politiques d'éducation et de formation pour anticiper les nouveaux besoins en main-d'œuvre qualifiée. Cette approche théorique globale converge avec l'analyse sectorielle menée par l'INRA dans le cadre de l'étude Ecophyto $R \mathcal{E} D^{16}$ qui porte sur les voies de réduction de l'usage des pesticides. Cette étude met en lumière un blocage du système de production agricole et agro-alimentaire dans son ensemble, blocage dans le sens où aucun acteur n'a intérêt à évoluer seul pour s'écarter de l'équilibre actuel, même si celui-ci est sous-optimal du point de vue de l'ensemble de la société. L'étude souligne aussi le besoin d'évolution des compétences et dessine un schéma d'apprentissage collectif passant par des réseaux d'expérimentation, des bases de données et une capitalisation des résultats et connaissances. Ces orientations sont aujourd'hui mises en œuvre dans le cadre d'un effort associant toutes les parties prenantes du plan Ecophyto 2018.

La PAC doit se saisir de cette dimension majeure, l'innovation, en s'attachant à créer des conditions qui la favorisent et l'encouragent non seulement au sein des exploitations agricoles, mais également dans les territoires et les filières.

Au niveau des exploitations agricoles, le remplacement des prix garantis et des aides liées aux produits par des paiements découplés ont levé une partie des obstacles à l'innovation à travers de nouvelles combinaisons de production, en brisant le lien entre les montants d'aides et les choix et niveaux de produits. Mais modifier les choix de variétés et de races, les assolements et les rotations, les pratiques et les itinéraires techniques est difficile car incertain, plus exigeant en compétences humaines et plus risqué d'un point de vue économique. La PAC a ici un rôle majeur à jouer, à un triple égard : d'abord, via la mise en place de mécanismes complets et efficaces de gestion des risques ; ensuite, en encourageant, via des aides ciblées temporaires, les démarches innovantes économes et écologiques ; enfin, en encourageant la recherche et la recherche-développement dans les dimensions génériques (par exemple, des variétés mieux adaptées à la sécheresse) et systémiques (par exemple, en combinant de façon coordonnée les aspects choix des variétés, techniques culturales, périodes $d$ 'intervention, etc.).

Sur les territoires, les échelles intermédiaires entre les exploitations agricoles et les régions sont reconnues comme des niveaux clés de gestion

16. J.-P. Butault, C.-A. Dedryver, C. Gary, L. Guichard, F. Jacquet, J.-M. Meynard, P. Nicot, M. Pitrat, R. Reau, B. Sauphanor, I. Savini et T. Volay, Ecophyto R\&D. Quelles voies pour réduire l'usage des pesticides ? Synthèse du rapport d'étude, Paris, INRA, 2010. 
des " agro-écosystèmes », que l'on s'intéresse à la biodiversité, à la gestion durable des résistances des cultures aux maladies et aux prédateurs, ou encore à la qualité des eaux. C'est en effet à cette échelle que se jouent de nombreuses régulations écologiques entre les organismes vivants ou que se forment quantité et qualité de l'eau. Appréhender ces échelles intermédiaires est le défi relevé par les scientifiques qui construisent le champ de l'agro-écologie, au croisement de l'écologie et de l'agronomie, en lien avec les sciences économiques, sociales et humaines. Il existe déjà des initiatives en ce domaine, en France et ailleurs (expériences de Vittel, de Munich ou de la baie de Chesapeake pour améliorer la qualité de l'eau potable, programme Centre national de la recherche scientifique [CNRS]-INRA " Agriculture et biodiversité » sur la zone atelier Plaine et Val de Sèvre, etc.). Pour rendre possible une transformation profonde de l'agriculture dans son ensemble, il faut démultiplier des zones de démonstration pertinentes d'un point de vue agro-écologique et socio-économique. La future PAC est l'occasion de mettre en place ces tremplins pour l'agriculture du futur. Au-delà des exploitations agricoles insérées dans des territoires, c'est l'ensemble des filières alimentaires qui est concerné. Il ne s'agira pas seulement de produire plus ; il faudra aussi limiter les pertes et gaspillages aux différents stades des filières, favoriser le recyclage des matières organiques, innover pour l'éco-conception et la diminution de l'empreinte environnementale des systèmes alimentaires - en ne limitant pas cette empreinte au seul carbone. Pour l'agriculture et les filières alimentaires, cette nécessaire ambition soulève la question de l'organisation optimale des activités dans les territoires conciliant ces différents objectifs environnementaux avec des objectifs de viabilité économique et de vie des territoires. En clair, peut-on sortir de la spécialisation excessive de certaines régions agricoles pour aller vers des combinaisons d'activités qui conjuguent performances économiques, sociales et environnementales ? Ici aussi, la PAC a un rôle à jouer via des dispositifs d'encouragement, d'accompagnement et d'évaluation des initiatives innovantes à une échelle dépassant celle de l'exploitation, à la fois verticalement (filières) et horizontalement (territoires).

Il ne s'agit pas pour autant de proposer un « grand soir » pour la PAC, faisant table rase du passé. Des évolutions positives ont été enregistrées depuis plusieurs années dans le cadre d'une démarche progressive qui tient compte des réalités politiques et vise à permettre l'adhésion du plus grand nombre. Mais à l'heure où les États membres et le Parlement européen vont entrer dans des négociations délicates, où chacun sortira sa calculatrice pour analyser s'il gagne ou perd à telle ou telle proposition dans une logique étroitement comptable, le plus important est de fixer un cap à la PAC. 
Le monde change, la PAC doit aussi changer. La route suivie depuis 20 ans de réformes, celle du découplage de la politique de soutien des revenus pour des raisons d'acceptation internationale et celle d'un ciblage augmenté sur l'environnement, doit se doubler d'une autre voie visant la stabilité, les biens publics et l'innovation. Il nous faut aujourd'hui construire cet avenir sur lequel les agriculteurs pourront s'engager. Un avenir plus sûr que celui qu'ils connaîtraient en empruntant l'impasse des avantages acquis. Un avenir plus sûr que la route du seul libéralisme qui, faute de moteurs suffisamment puissants, laisserait beaucoup de territoires sur le bord de la route. Un avenir plus sûr que la voie escarpée d'une PAC limitée aux seules préoccupations environnementales, qui laisserait aussi un grand nombre d'agriculteurs sur le côté.

MOTS CLÉS

Agriculture

PAC

Union européenne

Environnement 